\title{
Neoaorta Reconstruction Using Both Femoral Veins in a Patient with Secondary Aortoenteric Fistula
}

\author{
Zia-ur-Rehman
}

\begin{abstract}
In situ reconstruction using femoral veins is emerging as one of the acceptable options for aortic reconstruction in patients with aortoduodenal fistula. We report a 35-year young male who presented with secondary aortoenteric fistula. His infected aortic graft was removed and was successfully managed by neo-aortic reconstruction, using both femoral veins in a 'pantaloon' fashion. He had smooth postoperative recovery and did not have graft re-infection. He did not show signs of chronic venous insufficiency on long-term follow-up.
\end{abstract}

Key Words: Aortoduodenal fistula, Femoral vein, In situ reconstruction.

\section{INTRODUCTION}

Aortoduodenal fistula (ADF), after abdominal aortic aneurysm repair is an infrequent complication. ${ }^{1}$ If untreated, it is invariably fatal. ${ }^{2}$ Extra-anatomical bypass after removal of infected graft is the conventional treatment. However, it is associated with limited patency, recurrent infection and repeated procedures. ${ }^{3}$ There is also the risk of aortic stump blowout. In situ reconstruction with femoral vein grafts has been suggested as an alternative with good results. ${ }^{2,4-6}$ Little is reported in local literature on this topic.

We present a patient who underwent successful neoaorta reconstruction using both femoral veins for treatment of secondary ADF.

\section{CASE REPORT}

A 35-year male, cigarette smoker, developed backache and hematuria in September 2012. He was worked up outside our hospital and underwent cystoscopy, following which he developed deep venous thrombosis of the left leg. During investigations, he was found to have protein $\mathrm{C}$ deficiency. On performing MRI lumbosacral region for back pain, he was also found to have saccular abdominal aortic aneurysm. He was referred to us for aneurysm repair, which was measuring more than $6.5 \mathrm{~cm}$. It was repaired with Dacron bifurcated aorto-bi-iliac graft $9 \times 18 \mathrm{~mm}$. It was inflammatory in nature and was eroding vertebral body posteriorly. Tissue culture of the inflamed wall did not grow any organism.

Department of Surgery, Aga Khan University Hospital,

Karachi, Pakistan

Correspondence: Dr. Zia-ur-Rehman, Section of Vascular

Surgery, Department of Surgery, Aga Khan University Hospital,

Karachi, Pakistan

E-mail: ziaur.rehman@aku.edu

Received: October 03, 2018; Accepted: January 28, 2019
Four months later, the patient presented again in the emergency room with right lower leg cellulitis. He was having a high-grade fever and it was associated with rigors and shivering. Calf and thigh muscles were tender with intact vascularity. He did not have any gastrointestinal symptoms and was tolerating diet well. He was started on intravenous antibiotics; to which, he responded well. His fever settled and tenderness decreased within the next 1-2 weeks. His blood culture grew Candida and E. coli. On the basis of culture results, he was started on intravenous antifungal medications.

CT abdomen with intravenous contrast in the same admission was performed to evaluate his aortic reconstruction. It showed secondary ADF, as both limbs of Dacron graft were passing through duodenum with surrounding air (Figure 1).

As he was young and nutritionally well preserved, the decision for in situ reconstruction was made. This was to be performed after excision of infected graft and bowel repair. Although, he had deep venous thrombosis of left lower leg but recent venous duplex scan showed patent femoral and popliteal veins. After ensuring

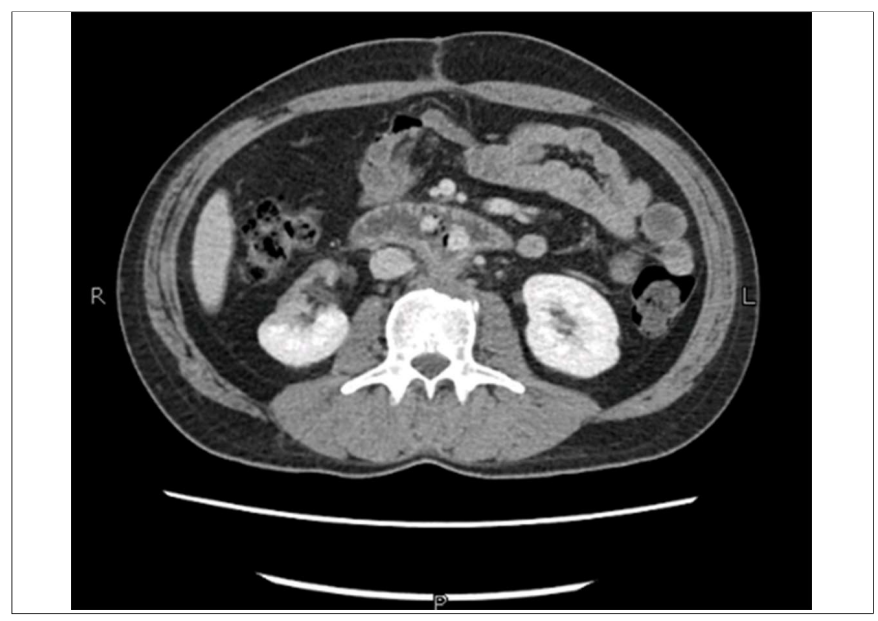

Figure 1: Both limbs of Dacron graft are passing through the duodenum. 


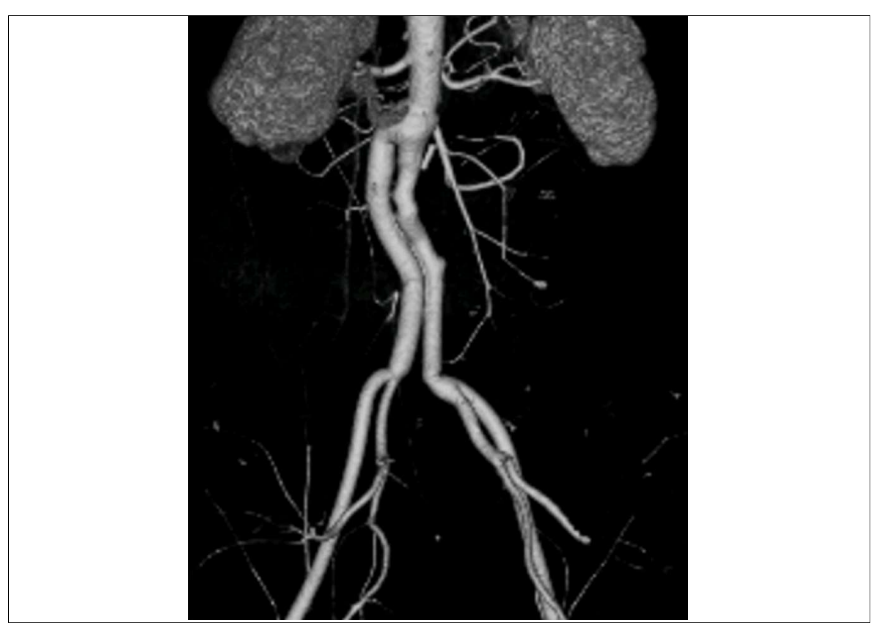

Figure 2: Follow-up CT scan after 1 year, showing patent graft.

patency, it was planned to use both femoral veins as 'pantaloon' graft. Risks and benefits were explained to the patient and family members in detail; to which, they consented. Femoral veins on both sides were dissected. Their tributaries were double ligated. The veins were dissected free from its junction from profunda femoral vein to the level of adductor canal and left in situ.

Then abdominal cavity was explored through midline via previous scar and supra coelic aortic control gained. There was intense, dense fibrosis in the retroperitoneum. The plane was created over the anterior aspect of Dacron graft, which was taken as a landmark to identify its junction with the aorta. The abdominal aorta below the renal arteries and both common iliac arteries were dissected and controlled. Infected graft was separated from the duodenum. A fistulous opening involving $50 \%$ of circumference between D3 to D4 was noted. It was primarily repaired. Femoral veins, which had been already dissected, were disconnected from both thighs. Both were everted and their valves excised. Proximal ends were split and joined in a 'pantaloon' fashion. Infected graft was removed; the aortic bed was debrided. End-to-end anastomoses of graft with the infrarenal aorta and both common iliac arteries were performed.

Greater omentum was placed between the duodenum and the venous graft. Venting gastrostomy and feeding jejunostomy were also created.

Patient tolerated the procedure. He was followed at one month, six months and then annually for 3 years. He is fine with no recurrent symptoms. He did not have significant limb swelling. CT scan at one year showed patent graft (Figure 2).

\section{DISCUSSION}

Femoral veins are good option for the reconstruction of neoaorta due to their size and high resistance to infection. ${ }^{4}$
The proximal end of the vein graft is 10 to $15 \mathrm{~mm}$ in diameter, and this allows comfortable anastomosis with the aorta to be constructed without getting any narrowing.

Venous insufficiency is a major concern after harvesting both femoral veins. Different authors have different experiences. Nevelsteen et al. reported minimal disability due to chronic venous insufficiency at a mean follow-up of 17 months. ${ }^{5}$ Clagett et al. found significant limb swelling, requiring compression stocking in only $15 \%$ of patients. ${ }^{6}$ One way to minimize this morbidity is to preserve profunda femoris vein during dissection. This patient did not have any significant limb swelling postoperatively and had no signs of chronic venous insufficiency on follow-up.

There has been evolution of technique of performing the in situ reconstruction from its initial description. ${ }^{4}$ One is eversion of vein and excising the valves under vision, as was performed in this case. There is always chance of incomplete valve lysis with valvulotome. The other modification is reinforcement of proximal anastomosis with tense fascia lata.

One of the disadvantages of this procedure is longer operative time. For this reason, one of the groups has suggested to perform it as staged procedure. On initial visit, harvest the femoral veins on both sides but keep them in situ. After 24 hours, on the second visit to the operation, perform excision of infected graft and neoaorta reconstruction.

In situ reconstruction with superficial femoral veins is an attractive and feasible alternative in the treatment of secondary ADF.

\section{REFERENCES}

1. Berger P, Vaartjes I, Moll FL, De Borst GJ, Blankensteijn JD, Bots ML. Cumulative incidence of graft infection after primary prosthetic aortic reconstruction in the endovascular era. Eur $J$ Vasc Endovasc Surg 2015; 49:581-5.

2. Yamamoto $\mathrm{Y}$, Igari $\mathrm{K}$, Toyofuku T, Kudo T, Inoue $\mathrm{Y}$. Late stent graft infection after the emergency endovascular repair of a secondary iliac artery-enteric fistula treated with graft removal and in situ aortic reconstruction using femoral veins. Ann Thorac Cardiovasc Surg 2017; 23:113-7.

3. Berger P, Moll FL. Aortic graft infections: Is there still a role for axillobi-femoral reconstruction? Semin Vasc Surg 2011; 24: 205-10.

4. Chung J, Clagett GP. Neoaortoiliac system (NAIS) procedure for the treatment of the infected aortic graft. Semin Vasc Surg $2011 ; 24: 220-6$.

5. Nevelsteen A, Lacroix H, Suy R. Autogenous reconstruction with the lower extremity deep veins: An alternative treatment of prosthetic infection after reconstructive surgery for aortoiliacdisease. J Vasc Surg 1995; 22:129-34.

6. Clagett GP, Bowers BL, Lopez-Viego MA, Rossi MB, Valentine RJ, Myers SI, et al. Creation of a neo-aorto-iliac system from lower extremity deep and superficial veins. Ann Surg 1993; 218: 239-48. 